\title{
Functional Outcomes of Primary Restoration of Elbow Flexion after Traumatic Brachial Plexus Palsy
}

\author{
MOHAMMAD S. ALSABBAHI, M.Sc.; MOHAMMAD H. ABDELALL, M.D.; AHMED M. ALY, M.D. and \\ MOHAMMAD R. AHMAD, M.D. \\ The Department of Plastic Surgery, Faculty of Medicine, Zagazig University, Zagazig
}

\begin{abstract}
Brachial plexus injury represents a wide spectrum of a complex presentation. The primary goal in salvaging upper extremity function in adult patients is restoration of elbow flexion, because it's highly needed in routine daily activity. So the goal of this study study is to assess different primary nerve procedures used in elbow flexion restoration after traumatic brachial plexus palsy.
\end{abstract}

Method: From April 2014 to May 2017, 22 patients with upper or total brachial plexus injury have had a primary procedure for elbow flexion restoration; nerve repair, nerve grafting and nerve transfer (double fascicular or intercostals) were used.

Results: Of the 22 patients (one patient had bilateral injury so 23 procedures were done). Double fascicular transfer in 7 limbs, 12 intercostal nerve transfers in 12 limbs and nerve grafts in 4 limbs. 16 cases $(69.6 \%)$ could achieve M3 or more.

Key Words: Brachial plexus palsy - Erb's palsy - Nerve graft - Nerve transfer - Intercostals.

\section{INTRODUCTION}

The injury of the brachial plexus is very difficult to apprehend, this is because it's complex anatomy and different types of injury [1]. The surgery for brachial plexus has evolved throughout the last 50 years with introduction of new techniques of microsurgery [2]. Restoring the elbow flexion has a higher priority in adults because of most of arm function depends on it [3].

Understanding the internal topography of the trunks and musculocutaneous nerve is essential for intraneural dissection and improving the outcome [4]. Within the upper trunk the motor fascicles for elbow are located medially [5]. The motor fibers to biceps, the sensory fibers and fibers to brachialis within the musculocutaneous nerve (MCN) are arranged in this order from lateral to medial [4].

Timing of repair is a crucial factor in predicting the outcome. There is wide agreement about the early intervention in the first 3 months (in cases where recovery is not expected) will improve the outcome [6]. In open injuries nerve surgery are indicated if no other life threatening injuries especially in sharp lacerations where healthy nerve endings can be traced, on the contrary in gunshots waiting for 3 to 4 months is advocated as spontaneous recovery is more likely $[\mathbf{7 , 8}$. In cases of root avulsion, a variety of intraplexal and extraplexal motor donors are available [9].

Oberlin et al., 1994 first described the transfer of motor ulnar fascicle of the ulnar nerve to the motor branch to the biceps [10]. The double fascicular transfer using extra fascicle of median nerve to brachialis showed better outcomes $[4,11]$. Seddon in 1963 first tried the intercostal nerves (ICNs) with the intention of restoring elbow flexion in a patient with complete root avulsion [12]. Chuang et al., 1992 and Malessy et al., 1993 recommended using three ICNs to recover elbow flexion function. $[\mathbf{1 3 , 1 4}]$. Later on reports by Asian surgeons for the use of the ICNs have led to popularization of this technique [15].

\section{PATIENTS AND METHODS}

This observational case series study investigated the primary techniques used in elbow flexion restoration at Zagazig University Hospitals, Plastic Surgery Department from April 2014 to May 2017. The study included 22 patients with traumatic brachial plexus injuries (23 limbs) as one patient had bilateral injury. Patients were managed accord- 
ing to their clinical diagnosis by either sural nerve cable grafts for root ruptures and sharp injuries, double fascicular transfer (DFT) for upper root avulsions (C5-6 $\pm \mathrm{C} 7)$, and intercostal nerves (ICNs) transfer for total root avulsions.

In the preoperative evaluation: All patients were subjected to full clinical evaluation including history of trauma and previous surgery, full motor and sensory examination, presence of Horner's and Tinel's signs. The British medical research council (MRC grade) was used for elbow movement assessment. Either CT myelography or MRI was used to complete diagnosis. EMG was done to confirm the diagnosis when needed only.

Operative procedures: The details of the different techniques used in this study are beyond the scope of this paper, but the general steps were as the following: General anesthesia was used without using muscle relaxant to allow intraoperative nerve stimulation. Magnification was achieved by $6 \mathrm{X}$ surgical loupes. In 18 cases a supraclavicular brachial plexus exploration (Fig. 1) was done to confirm root avulsion or root rupture. In 2 cases axillary exploration for sharp laceration was used, and in 3 cases direct nerve transfer was preferred from the start to avoid vascular injuries with disturbed anatomy.

In cases of nerve transfer (17 cases) medial arm incision was used to access the motor nerves to biceps and brachialis for more distal neurotization by either the double fascicular transfer from median and ulnar or by the ICNs (Figs. 2,3). All neural anastomosis was done using a perineural repair with $9 / 0$ or $10 / 0$ nylon sutures and fibrin glue. Other techniques were used for shoulder and hand if needed (spinal accessory to suprascapular nerve transfer and cross $\mathrm{C} 7$ to median nerve transfer via vascularized ulnar graft respectively).

Immobilization in arm to chest position for 1 month then physiotherapy starts by galvanic current stimulation of affected muscles and physical therapy to affected joints. Follow-up continued monthly with a mean follow up time was 19.5 \pm 7.2 months (the shortest was 10 and longest was 36).

Data were collected and analyzed using SPSS 22 software.

\section{RESULTS}

The patient demographics showed that 19 $(86.4 \%)$ cases were males and $3(13.6 \%)$ were females. 15 (68.2\%) patients were from Sharkia and $7(31.8 \%)$ patients were from other governorates. Affected side was right in $12+1(56.5 \%)$ and left $9+1(43.5 \%)$ as one patient had bilateral injury. The type of trauma was road traffic accidents in $17(77.3 \%)$ patients, fall from height in $3(13.6 \%)$ patients and sharp injury in $2(9 \%)$ patients. The mean age was $26.5 \pm 14.6$ (the youngest was 2 years and the oldest was 59). The mean time before operation was $5.17 \pm 2.99$ (the shortest was two weeks and the longest was 12 months).

The types of primary interventions that has been done were double fascicular transfer in 7 $(30.4 \%)$ cases, intercostal nerve transfer in 12 (52.4) cases and sural nerve cable grafts in 4 $(17.4 \%)$ cases (Table 1). The mean operative time was $4.04 \pm 0.88$ hours (shortest was 2.5 hours and longest was 6 hours). This was the total operative time including associated procedures for shoulder and/or hand. The mean follow-up time was 19.5 \pm 7.2 months (the shortest was 10 and longest was 36).

Most of the patients had showed progressive improvement of elbow flexion function during the follow up period regardless to the type of the procedure that was done (Figs. 5,6). Wilcoxon Signed Ranks Test comparing the pre and post-operative MRC score showed overall significant improvement $(p$-value $=0.000)$. However, the selective postoperative MRC scores showed these results: 16 limbs (69.6\%) that had a score of M3 or more and $5(21.7 \%)$ limbs that had M1 or M2 scores and only $2(8.7 \%)$ limbs showed no improvement at all (M0). The details are summarized in (Fig. 4).

Using Spearman Correlation, there was no significant relationship between the age and the postoperative MRC score ( $p$-value $=0.290)$.

Table (1): Types of procedures.

\begin{tabular}{ccc}
\hline & \multicolumn{2}{c}{ Types of Interventions } \\
\cline { 2 - 3 } & Frequency & Percent \\
\hline DFT & 7 & 30.4 \\
ICN & 12 & 52.2 \\
Graft & 4 & 17.4 \\
& & 100.0 \\
\hline
\end{tabular}

DFT $=$ Double fascicular transfer

$\mathrm{ICN}=$ Intercostal nerve transfer. 


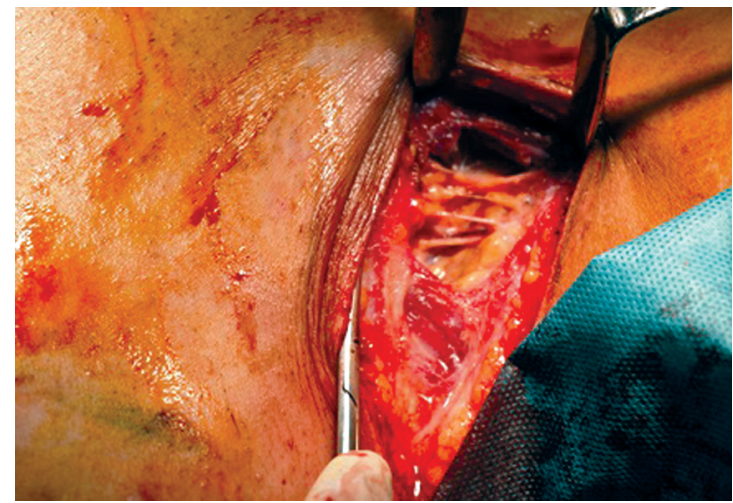

Fig. (1): Supraclavicular exploration showing the supraclavicular nerves in the subcutaneous tissue.

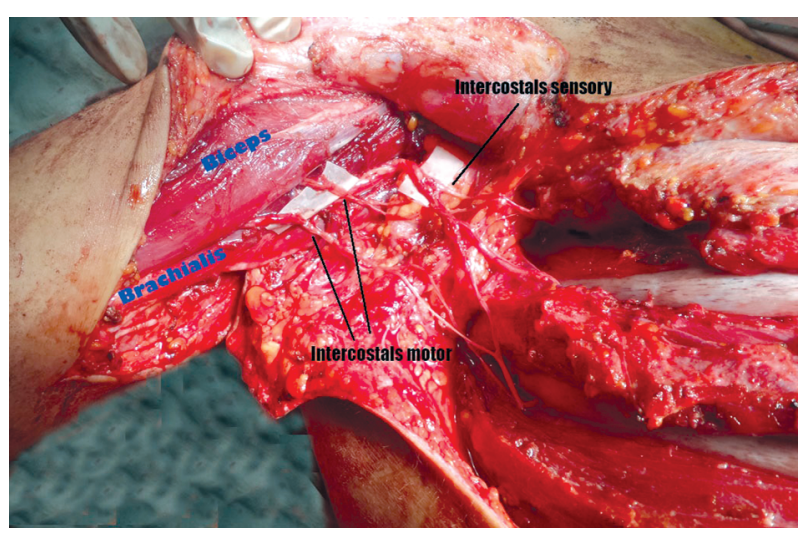

Fig. (3): $3^{\text {rd }}, 4^{\text {th }}, 5^{\text {th }}$ and $6^{\text {th }}$ intercostal nerve transfer to the motor branches of the biceps and brachialis.

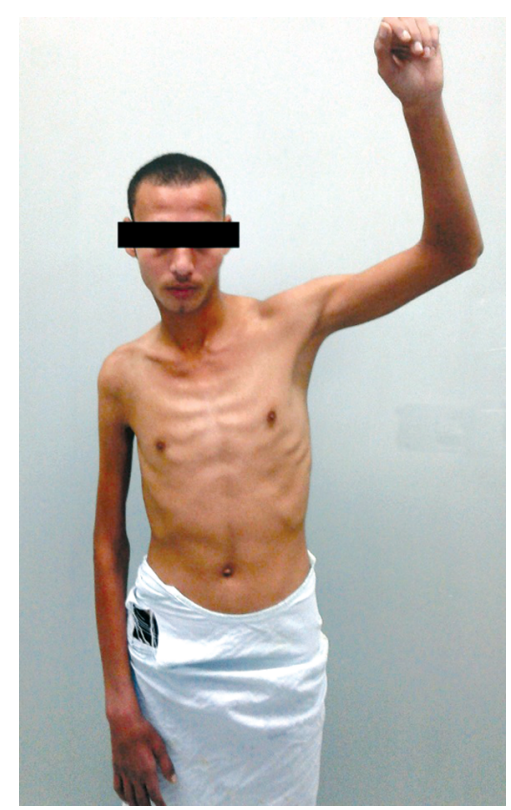

Fig. (5): Right total roots injury in a 22 years old male.

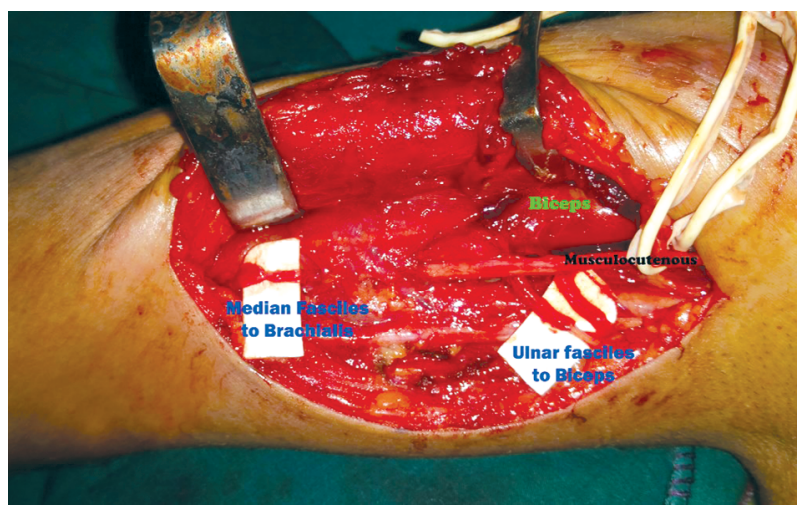

Fig. (2): The transfer of ulnar and median fascicles to the biceps and brachialis branches of the MCN respectively.

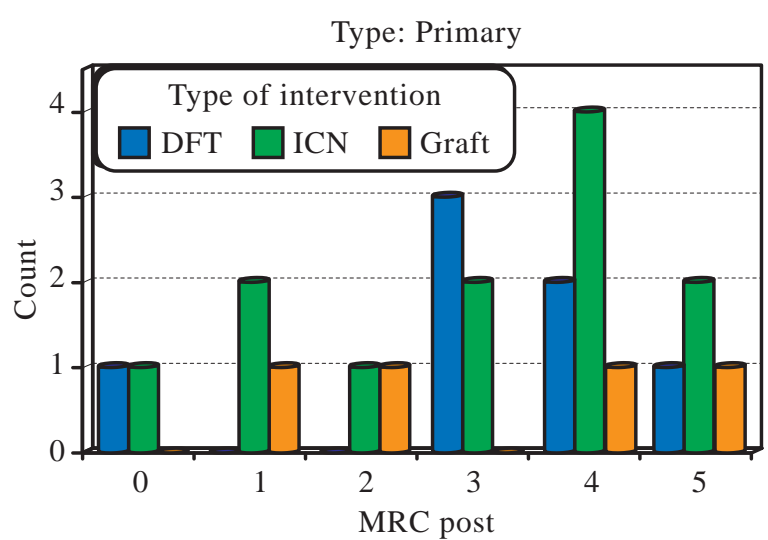

Fig. (4): Cluster bar chart shows the postoperative MRC score for each selected primary intervention.

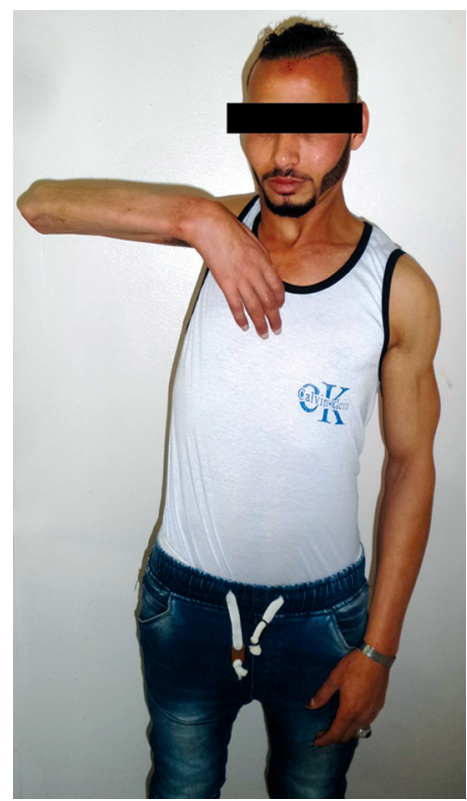

Fig. (6): Results after Intercostal nerve transfer. 


\section{DISCUSSION}

Restoration of elbow flexion represents the first priority in traumatic brachial plexus cases as it's important for every day activity [16,17]. Achieving this through nerve surgery has more superior results $[18,19]$. In cases of upper brachial plexus injury $(\mathrm{C} 5, \mathrm{C} 6 \pm \mathrm{C} 7)$ the use of intraplexal motor donors is more physiologic, thus enhancing the chances for recovery [9].

In this study; 16 limbs $(69.6 \%)$ that had a score of M3 or more and 5 (21.7\%) limbs that had M1 or M2 scores and only $2(8.7 \%)$ limbs showed no improvement at all (M0).

Two series reported the success of DFT for elbow restoration. One reported achieving MRC grade $4 / 5$ in 4 out of 6 patients and the other reported achieving 4/5 in all 6 patients. The two studies did not report any sensory or motor deficit of both median and ulnar nerves as a donor $[4,20]$.

Another series investigated the better outcome achieved by double fasicular transfer over the Oberlin's procedure and showed significant difference between the two groups [11].

In this series 6 cases out of $7(85.7 \%)$ could achieve M3 or more showing that DFT is a successful method of restoring elbow flexion which agrees with other results from similar studies.

In global plexopathy, lack of intraplexal donors and a paucity of motor donors have yielded inferior results to intraplexal transfers. The ICNs are one of the heavily used transfers in elbow restoration. In a large series (718 transfers of ICNs) for elbow flexion, extension, and shoulder function. They achieved good elbow flexion in $72 \%$ of cases. It was found that the use of more nerves yields better results [21]. Another study showed the regaining of M3 or more elbow function after ICNs to MCN transfer in 5 out of 8 patients (62.5\%) [2].

In our series 8 out of $12(66.6 \%)$ patients could achieve M3 or more denoting the high efficacy of ICNs transfer for restoring elbow flexion in cases where no intraplexal donors available.

In this study we found no significant correlation between age and outcome which disagrees with the results of the other studies $[15,16,19,20]$. This may be explained by the fewer number of patients at extremes of age (most of the patients were middle age group).
Conclusion: The results of this study support the worldwide agreement about the advantage of primary repair in traumatic brachial plexus cases and shows that primary surgical intervention provides an efficient solution, with marked improvement of the quality of life and patient satisfaction.

\section{REFERENCES}

1- Matsuyama T., Okuchi K., Akahane M., Inada Y. and Murao Y.: Clinical analysis of 16 patients with brachial plexus injury. Neurologia Medico-chirurgica. 42 (3): 11421, Discussion, 22, 2002.

2- Ahmad M.R.: Early post-operative results after repair of traumatic brachial plexus palsy. Turkish Neurosurgery, 23 (1): 1-9, 2013.

3- Terzis J.K. and Barbitsioti A.: Primary restoration of elbow flexion in adult post-traumatic plexopathy patients. Journal of Plastic, Reconstructive \& Aesthetic Surgery: JPRAS, 65 (1): 72-84, 2012.

4- Mackinnon S.E., Novak C.B., Myckatyn T.M. and Tung T.H.: Results of reinnervation of the biceps and brachialis muscles with a double fascicular transfer for elbow flexion. J. Hand Surg. Am., 30 (5): 978-85, 2005.

5- Tung T.H. and Moore A.E.: Brachial Plexus Injuries. In: Mackinnon SE, editor. Nerve Surgery New York: Thieme, p. 339-485, 2015.

6- Bhandari P. and Bhatoe H.: Is fascicular selection by nerve stimulation techniques a necessity in selective nerve transfers targeted at restoration of elbow flexion in upper brachial plexus injuries? Indian Journal of Neurotrauma, 8 (2): 99-104, 2011.

7- Quinones-Hinojosa A. Schmidek and Sweet: Operative Neurosurgical Techniques E-Book: Indications, Methods and Results (Expert Consult - Online and Print): Elsevier Health Sciences, 2012.

8- Fox I.K. and Mackinnon S.E.: Adult peripheral nerve disorders: Nerve entrapment, repair, transfer, and brachial plexus disorders. Plastic and Reconstructive Surgery, 127 (5): $105 \mathrm{e}-18 \mathrm{e}, 2011$.

9- El-Hoseny A.Y., Ahmad M.R. and Hussein Y.: New proposed prevertebral approach for turned on normal contralateral C7 as a donor for avulsed brachial plexus. Journal of American Science, 7 (4): 661-8, 2012.

10- Oberlin C., Beal D., Leechavengvongs S., Salon A., Dauge M.C. and Sarcy J.J.: Nerve transfer to biceps muscle using a part of ulnar nerve for C5-C6 avulsion of the brachial plexus: Anatomical study and report of four cases. J. Hand Surg. Am., 19 (2): 232-7, 1994.

11- Ahmed M.R. and Aly A.M.: Biceps Neurotization Versus Biceps and Brachialis Neurotization in Brachial Plexus Injury: Comparative Study. Egypt, J. Plast. Reconstr. Surg., 41 (1): 111-6, 2017.

12- Seddon H.J.: Nerve grafting. Annals of the Royal College of Surgeons of England, 32: 269-80, 1963.

13- Chuang D.C., Yeh M.C. and Wei F.C.: Intercostal nerve transfer of the musculocutaneous nerve in avulsed brachial 
plexus injuries: Evaluation of 66 patients. J. Hand Surg. Am., 17 (5): 822-8, 1992.

14- Malessy M.J., Van Dijk J.G. and Thomeer R.T.: Respiration-related activity in the biceps brachii muscle after intercostal-musculocutaneous nerve transfer. Clinical Neurology and Neurosurgery, 95 Suppl: S95-102, 1993.

15- Socolovsky M. and Paez M.D.: A literature review of intercostal-to-musculocutaneous-nerve transfers in brachial plexus injury patients: Does body mass index influence results in Eastern versus Western countries? Surgical Neurology International, 4: 152, 2013.

16- Ray W.Z., Pet M.A., Yee A. and Mackinnon S.E.: Double fascicular nerve transfer to the biceps and brachialis muscles after brachial plexus injury: Clinical outcomes in a series of 29 cases. Journal of Neurosurgery, 114 (6): 1520-8, 2011.

17- Faglioni W., Jr., Siqueira M.G., Martins R.S., Heise C.O. and Foroni L.: The epidemiology of adult traumatic brachial plexus lesions in a large metropolis. Acta. Neurochirurgica, 156 (5): 1025-8, 2014.

18- Tung T.H. and Mackinnon S.E.: Nerve transfers: Indications, techniques, and outcomes. J. Hand Surg. Am., 35 (2): 332-41, 2010.

19- Estrella E.P.: Functional outcome of nerve transfers for upper-type brachial plexus injuries. Journal of plastic, reconstructive \& aesthetic surgery: JPRAS, 64 (8): 1007 13, 2011.

20- Liverneaux P.A., Diaz L.C., Beaulieu J.Y., Durand S. and Oberlin C.: Preliminary results of double nerve transfer to restore elbow flexion in upper type brachial plexus palsies. Plastic and Reconstructive Surgery, 117 (3): 9159, 2006.

21- Terzis J.K. and Kostopoulos V.K.: The surgical treatment of brachial plexus injuries in adults. Plastic and Reconstructive Surgery, 119 (4): 73e-92e, 2007. 